European Federation of Societies for Ultrasound in Medicine and Biology

\title{
Ultrasound is unique
}

\author{
by EFSUMB President Odd Helge Gilja
}

Dear EFSUMB member,

Ultrasound is unique!

Its uniqueness is reflected in its enormous capacity to do great things for the patients. Not only can ultrasound in the right hands diagnose a vast variety of diseases, but it can also be used for therapy. Not only can ultrasound provide very high temporal and spatial resolution on high-end scanners in hospital departments, but mobile ultrasound can also be applied in the battle field or in the helicopter for point-of-care medicine and telemedicine. Ultrasound is indeed unique. Moreover, its uniqueness outperforms other radiology methods in its versatility and huge span of applications.

Therefore, EFSUMB has an important mission to disseminate information and competence, thus, building a European Ultrasound Community. EFSUMB now has more than 20000 members and is the biggest ultrasound federation in the world of ultrasound in medicine and biology. In the following, I will point at some good opportunities for you to further build competence in ultrasound.

\section{The 29th Euroson Congress}

The EUROSON congress 2017 will be arranged by the Turkish Ultrasound Society (TUDS) and take place 22 - 25 September in Ljubljana, Slovenia. This will be the EFSUMB highlight of the year with an exciting program for science and education in the field of ultrasound. This Euroson congress will be a vibrant, interdisciplinary meeting place for students, doctors, engineers, professors, sonographers, industry and all stakeholders of ultrasound. It will focus on new developments in science and education and renowned speakers from all across Europe will provide state-of-the-art lectures and live demonstrations. There will be an Ultrasound Learning Center (ULC) for hands-on training where students and young doctors can experience supervision and guidance from experts in various fields. A dedicated student program will enhance the training outcome for young students, enabling a good start to their future careers.

\section{Euroson Schools and Endorsed courses}

EFSUMB has now conducted over 100 Euroson Schools all over Europe, thus contributing greatly to the advancement of ultrasound knowledge and skills. For 2017 there are 8 Euroson Schools in the pipeline covering aspects like MSK, vascular, rheumatology, CEUS applications, paediatrics, chest and hepatology. In addition, there are 8 EFSUMB-endorsed courses planned for 2017, see more at http://www.efsumb. org .

\section{Ultrasound Learning Centres (ULC) across Europe}

EFSUMB Ultrasound Learning Centres (ULC) are established in 7 renowned ultrasound locations around Europe: Timisoara, Neuruppin, Cluj-Napoca, Bergen, Madrid, Pavia and Zurich. At these excellent teaching sites, participants can spend a period of time to learn and to improve their knowledge in various applications of ultrasound. The teaching language is English if participants are from outside the host country. You may apply directly to the ULC Director and this information is located at http:// www.efsumb.org/euroson-sch/eul-centres01.asp.

\section{EFSUMB Webinar}

Our series of webinars is a flexible and popular way to achieve increased knowledge on various ultrasound topics. The first EFSUMB webinar was on CEUS of the liver, then followed by a webinar on non-liver CEUS, subsequently a webinar on CEUS in the evaluation of treatment procedures, and the previous webinar was on CEUS beyond Europe - The Chinese experience. All these lectures can still be viewed in our Webinararchive at http://www.efsumb.org/education/ed-webinar-archive.asp along with information about the last webinar on LIRADS 19. January 1800 CET.

\section{EFSUMB guidelines and clinical recommendations}

The world's first ever guidelines on gastrointestinal ultrasound (GIUS) was released by EFSUMB last year. These clinical recommendations and guidelines on methodology were published online in EJU September 2016 and are soon to appear in print with an editorial by Seitz, Ødegaard and Lutz. An EFSUMB Task Force Group (TFG) with experts from all over Europe is continuously working to make more GIUS guidelines and the next topic will be ultrasound in IBD. Other GIUS guidelines are also in pipeline: Inflammatory conditions, transrectal/perineal US, miscellaneous, Upper GI / functional US.

Existing EFSUMB guidelines are continuously being updated. The EFSUMB guidelines 
on liver elastography are soon ready for publication in short and long versions. Furthermore, an update on non-liver CEUS guidelines is also planned for release in 2017. The work on non-liver elastography has started and publication is estimated for 2018.

\section{EFSUMB Student}

\section{Committee}

EFSUMB has included students more closely under the EFSUMB umbrella by establishing a student committee. The Student Com- mittee, which at present is an interim committee, will be finally elected in 2017 at the Euroson congress. Some of the main tasks of ESC are to stimulate integration of ultrasound teaching and training in medical education of students and to establish quality standards. Furthermore, ESC shall plan a program for student activities and teaching at the EUROSON congresses in close collaboration with the local organisers.

Finally, I want to extend my dearest appreciation to all EFSUMB officers for their great work to promote ultrasound in Europe. In particular, I want to give my warmest thanks to our General Secretary Lynne Rudd for all her great work for EFSUMB.

\section{Bergen, January 2017,} Odd Helge Gilja

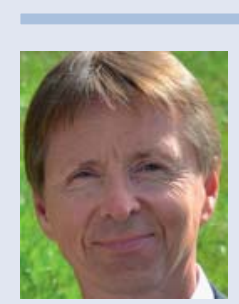

Odd Helge Gilja 